\title{
A preconditioning-based analysis for a Bakhvalov-type mesh
}

\author{
Thái Anh Nhan ${ }^{1} \quad$ Vinh Quang Mai ${ }^{2}$
}

(Received 23 December 2020; revised 14 December 2021)

\begin{abstract}
A new preconditioning-based parameter-uniform convergence analysis is presented for one-dimensional singularly perturbed convectiondiffusion problems discretized by an upwind difference scheme on a Bakhvalov-type mesh. The proof utilizes the classical convergence principle: uniform stability and uniform consistency imply uniform convergence, which can only be used after applying an appropriate preconditioner to the discrete operator.
\end{abstract}

\section{Contents}

1 Introduction

2 Upwind scheme on a Bakhvalov-type mesh

DoI:10.21914/anziamj.v62.16093, (C) Austral. Mathematical Soc. 2022. Published 2022-02-07, as part of the Proceedings of the 19th Biennial Computational Techniques and Applications Conference. ISSN 1445-8810. (Print two pages per sheet of paper.) Copies of this article must not be made otherwise available on the internet; instead link directly to the DOI for this article. 


\section{Introduction}

The purpose of this article is to provide a new theoretical analysis for the one-dimentional singularly perturbed convection-diffusion problem

$$
\mathcal{L} u:=-\varepsilon u^{\prime \prime}-b(x) u^{\prime}+c(x) u=f(x), \quad x \in(0,1), \quad u(0)=u(1)=0,
$$

where $\varepsilon$ is a positive perturbation parameter, $0<\varepsilon \leqslant 1$. We assume that the functions $b, c$ and $f$ are sufficiently smooth, and that

$$
b(x) \geqslant \beta>0, \quad c(x) \geqslant 0 \quad \text { for } x \in I:=[0,1] .
$$

When $\varepsilon$ is small, the problem (1) is convection-dominated and an exponential boundary layer typically arises when the flow (presented by the convection term $b(x)>0$ ) travels towards the boundary. Then, the boundary value problem (1) has a unique solution $\mathfrak{u} \in \mathrm{C}^{2}(\mathrm{I})$.

Because of the presence of such exponential layers, special numerical methods are derived to meaningfully resolve the layers and to achieve parameter-robust convergence (or $\varepsilon$-uniform convergence). The use of layer-adapted meshes, in conjuntion with either finite-difference or finite-element discretizations, is one of the most frequently used approaches to achieve the goal. In 1969, Bakhvalov [1] introduced the first layer-fitted mesh by applying the inverse of the exponential-layer function into its mesh-generating function. About two decades later, Shishkin [12] proposed the piecewise-uniform mesh which received much interest due to its simplicity in construction and analysis. Nevertheless, as a trade-off, the convergence rate of the Shishkin mesh is usually sub-optimal compared to that of the Bakhvalov mesh. For further important concepts and advances in the field of numerical analysis of singular perturbation problems, we refer the reader to the works by Linß [3], Roos et al. [11], Roos and Stynes [10], and Stynes [13]. 
Even when layer-adapted meshes are used, error analyses of finite-difference methods for problem (1) are still challenging. This is because the derivatives of the solution behave like $\mathcal{O}\left(\varepsilon^{-k}\right)$, where $k$ is some positive integer. Furthermore, the truncation errors of an upwind difference scheme discretized on the Shishkin mesh are not $\mathcal{E}$-uniformly consistent, and of order $\mathcal{O}\left(\varepsilon^{-1} \mathrm{~N}^{-1} \ln \mathrm{N}\right)$ where $\mathbf{N}$ is the discretization parameter $[6,14$, for numerical observations of this phenomenon]. Because of these issues, special techniques are devised to prove $\varepsilon$-uniform convergence for finite-difference methods on layer-adapted meshes. These include the hybrid-stability approach [4], truncation-error and barrier functions [7, 8], and the grid transformation [5, Chapter 7]. Recently, another method developed for proving uniform convergence on the Shishkin mesh is the preconditioning technique to enable the classical principle: " $\varepsilon$ uniform stability and $\mathcal{\varepsilon}$-uniform consistency imply $\mathcal{E}$-uniform convergence". This idea was first introduced by Vulanović and Nhan [14] and extended further by Nhan et al. [6] and Vulanović and Nhan [15] to handle hybrid higher-order finite-difference schemes, but only on the piecewise-uniform Shishkin mesh.

The goal of this article is to show that it is possible to generalize the preconditioning-driven analysis to an exponentially graded Bakhvalov-type mesh. In particular, our motivation comes from the fact that this new approach has proven its salient advantage over the aforementioned proofs. As shown by Vulanović and Nhan [15], an almost-third-order difference scheme can be only analysed by the preconditioning. We emphasise that our intended contribution is not the main uniform convergence theorem (already proven by other methods), but rather it is the novel analysis which makes use of the preconditioning approach for a Bakhvalov-type mesh. Our result might be employed to analyse more complicated higher-order schemes, similar to Vulanović and Nhan [15] but on Bakhvalov-type meshes.

In the next section, we describe an upwind discretization on a Bakhvalov-type mesh. Section 3 introduces an appropriate preconditioner to scale the discretized system and obtain the $\varepsilon$-uniform stability. Finally, the preconditioned consistency error is analysed and the uniform convergence result is derived. 


\section{Upwind scheme on a Bakhvalov-type mesh}

The following decomposition of $\boldsymbol{u}$ is often used in the error analysis of numerical methods for problem (1) [3, Theorem 3.48]:

$$
\begin{aligned}
u(x) & =s(x)+y(x), \\
\left|s^{(k)}(x)\right| \leqslant C\left(1+\varepsilon^{2-k}\right), \quad\left|y^{(k)}(x)\right| & \leqslant C \varepsilon^{-k} e^{-\beta x / \varepsilon}, \quad x \in I, \quad k=0,1,2,3,
\end{aligned}
$$

where $\mathrm{C}$ is a positive generic constant independent of $\varepsilon$ and $\mathrm{N}$. Moreover, the layer component $\mathrm{y}$ satisfies a homogeneous differential equation:

$$
\mathcal{L} y(x)=0, \quad x \in(0,1) .
$$

Let $I^{N}$ denote an arbitrary mesh with mesh points $x_{i}, i=0,1, \ldots, N$, such that $0=x_{0}<x_{1}<\cdots<x_{N}=1$. Let $h_{i}=x_{i}-x_{i-1}, i=1,2, \ldots, N$, be the mesh-step sizes and let $\bar{h}_{i}=\left(h_{i}+h_{i+1}\right) / 2$. Mesh functions on $I^{N}$ are denoted by, for example, $W^{N}=\left(W_{i}^{N}\right), u^{N}=\left(U_{i}^{N}\right)$. If $g$ is a function defined on I, then $g_{i}:=g\left(x_{i}\right)$, and $g_{i}^{N}:=g^{N}\left(x_{i}\right)$ for the corresponding mesh function. We use the maximum norm of $W^{N},\left\|W^{N}\right\|=\max _{0 \leqslant i \leqslant N}\left|W_{i}^{N}\right|$. The matrix norm induced by the maximum vector norm is also denoted by $\|\cdot\|$.

The upwind finite-difference scheme is used to discretize the problem (1) on $\mathrm{I}^{\mathrm{N}}$ :

$$
\begin{gathered}
u_{0}^{N}=0, \quad u_{N}^{N}=0, \\
\mathcal{L}^{N} u_{i}^{N}:=-\varepsilon D^{\prime \prime} u_{i}^{N}-b_{i} D^{+} u_{i}^{N}+c_{i} u_{i}^{N}=f_{i}, \quad i=1,2, \ldots, N-1,
\end{gathered}
$$

where

$$
\begin{aligned}
& \mathrm{D}^{\prime \prime} \mathrm{U}_{i}^{\mathrm{N}}=\frac{1}{\overline{\mathrm{h}}_{i}}\left(\mathrm{D}^{+} \mathrm{U}_{i}^{\mathrm{N}}-\mathrm{D}^{-} \mathrm{U}_{i}^{\mathrm{N}}\right), \\
& \mathrm{D}^{+} \mathrm{u}_{i}^{\mathrm{N}}=\frac{\mathrm{U}_{i+1}^{\mathrm{N}}-\mathrm{U}_{\mathrm{i}}^{\mathrm{N}}}{\mathrm{h}_{\mathrm{i}+1}}, \quad \mathrm{D}^{-} \mathrm{U}_{i}^{\mathrm{N}}=\frac{\mathrm{U}_{i}^{\mathrm{N}}-\mathrm{U}_{i-1}^{\mathrm{N}}}{\mathrm{h}_{i}} .
\end{aligned}
$$




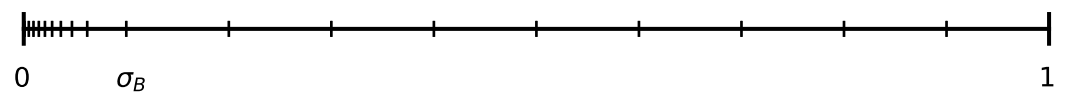

Figure 1: The Bakhvalov-type mesh defined by (6) and (7) for one-dimensional convection-diffusion problems (1).

We are interested in the Bakhvalov-type mesh introduced by Boglaev [2]:

$$
x_{i}= \begin{cases}\varepsilon \phi_{B}\left(t_{i}\right), & i=0,1, \ldots, J, \\ \sigma_{B}+2\left(1-\sigma_{B}\right)\left(t_{i}-1 / 2\right), & i=J+1, J+2, \ldots, N,\end{cases}
$$

with $\phi_{\mathrm{B}}(\mathrm{t}):=-\ln [1-2(1-\varepsilon) t], t_{i}=i / N, J=N / 2$, and transition point defined by

$$
\sigma_{\mathrm{B}}:=\varepsilon \ln (1 / \varepsilon)=x_{\mathrm{J}} .
$$

The mesh, plotted in Figure 1, is gradually graded in the layer region with $h_{i-1} \leqslant h_{i}, i=2, \ldots, J$, and is equally spaced with the step size $H:=h_{i}$, $i=J+1, \ldots, N$. This Bakhvalov-type mesh shares the following characteristics with the original Bakhvalov mesh: a logarithmic function to generate the points in the layer, and a transition point $(7)$ of the order $\mathcal{O}(-\varepsilon \ln \varepsilon)$. Furthermore, the last mesh step, $h_{J}:=\varepsilon \ln (1+2(1-\varepsilon) /(\varepsilon N))$, in the layer region tends to zero as $\varepsilon \rightarrow 0$; but more slowly than that of the Shishkin-type meshes (in the sense described by Roos and Linß [9]) due to the logarithmic factor. This is why the analysis for the Bakhvalov-type meshes is usually more delicate than the Shishkin-type meshes [8].

Lemma 1. The mesh widths in the layer regions of the Bakhvalov-type mesh defined by (6) and (7) satisfy

$$
h_{i-1} \leqslant h_{i} \leqslant C N^{-1}, \quad i=2,3, \ldots, J,
$$

and in particular

$$
h_{i} \leqslant \varepsilon, \quad i=1,2, \ldots, J-1 .
$$


Furthermore,

$$
e^{-\beta x_{J-1} / \varepsilon}=\left(\varepsilon+2(1-\varepsilon) N^{-1}\right)^{\beta} \leqslant\left(\varepsilon+2 N^{-1}\right)^{\beta}
$$

and

$$
e^{-\beta x_{\mathrm{J}} / \varepsilon}=\varepsilon^{\beta} .
$$

Proof: By the definition of $\phi_{\mathrm{B}}(\mathrm{t})$, we have $\phi_{\mathrm{B}}^{\prime}(\mathrm{t})=2(1-\varepsilon) /[1-2(1-\varepsilon) t]$, thus $\phi_{\mathrm{B}}(t)$ is monotonically increasing for $t \in[0,1 / 2]$. Therefore, $h_{i-1} \leqslant h_{i}$ $i=2,3, \ldots, J-1$, and

$$
h_{J}=\varepsilon \int_{t_{J}-1}^{t_{J}} \phi_{B}^{\prime}(s) d s \leqslant \frac{\varepsilon}{N} \phi_{B}^{\prime}\left(t_{J}\right)=\frac{\varepsilon}{N} \cdot \frac{2(1-\varepsilon)}{1-2(1-\varepsilon) t_{J}} \leqslant \frac{\varepsilon}{N} \cdot \frac{2}{\varepsilon}=2 N^{-1},
$$

which gives (8).

For the bound in (9), we have

$$
\begin{aligned}
h_{i} & =\varepsilon \int_{t_{i-1}}^{t_{i}} \phi_{B}^{\prime}(s) d s \leqslant \frac{\varepsilon}{N} \max _{t \in\left[t_{i-1}, t_{i}\right]} \phi_{B}^{\prime}(t)=\frac{\varepsilon}{N} \cdot \frac{2(1-\varepsilon)}{1-2(1-\varepsilon) t_{i}} \\
& \leqslant \frac{\varepsilon}{N} \cdot \frac{2}{1 /(1-\varepsilon)-2 t_{J-1}} \leqslant \frac{\varepsilon}{N} \cdot N=\varepsilon .
\end{aligned}
$$

We use the definitions of $x_{J-1}$ in (6) and $x_{J}$ in (7) to get the inequality (10) and the equality (11).

When the upwind scheme (5) is discretized on the Bakhvalov-type mesh, the linear system in matrix form is

$$
A_{N} U^{N}=f^{N}
$$

where $A_{N}=\left[a_{i j}\right]$ is a tridiagonal matrix with $a_{00}=1$ and $a_{N N}=1$ being the only nonzero elements in the 0 th and $\mathrm{N}$ th rows, respectively, and where 
$f^{N}=\left[0, f_{1}, f_{2}, \ldots, f_{N-1}, 0\right]^{\top}$. Let the entries of $A_{N}$ be denoted by $a_{i, j}$, the nonzero ones being

$$
\begin{aligned}
& \mathrm{l}_{i}:=\mathrm{a}_{i-1, i}= \begin{cases}-\frac{\varepsilon}{\bar{h}_{i} h_{i}}, & 1 \leqslant i \leqslant J, \\
-\frac{\varepsilon}{\mathrm{H}^{2}}, & J+1 \leqslant i \leqslant N-1,\end{cases} \\
& r_{i}:=a_{i, i+1}= \begin{cases}-\frac{\varepsilon}{\overline{h_{i}} h_{i+1}}-\frac{b_{i}}{h_{i+1}}, & 1 \leqslant i \leqslant J-1, \\
-\frac{\varepsilon}{\bar{h}_{i} H}-\frac{b_{i}}{H}, & i=J, \\
-\frac{\varepsilon}{H^{2}}-\frac{b_{i}}{H}, & J+1 \leqslant i \leqslant N-1,\end{cases}
\end{aligned}
$$

and

$$
d_{i}:=a_{i i}= \begin{cases}1, & i=0, \\ -l_{i}-r_{i}+c_{i}, & 1 \leqslant i \leqslant N-1 \\ 1, & i=N .\end{cases}
$$

\section{$3 \quad$ Preconditioning and $\varepsilon$-uniform stability}

The goal of this section is to precondition the discrete systems (5) in such a way that the $\mathcal{\varepsilon}$-uniform stability is retained; and simultaneously, the modified consistency errors can be proven to be convergent uniformly in $\varepsilon$.

Let $M=\operatorname{diag}\left(m_{0}, m_{1}, \ldots, m_{N}\right)$ be a diagonal matrix with the entries

$$
\mathrm{m}_{0}=1, \quad \mathrm{~m}_{\mathrm{i}}=\frac{\overline{\mathrm{h}}_{\mathrm{i}}}{\mathrm{H}}, \quad \mathrm{i}=1, \ldots, \mathrm{J}, \quad \text { and } \quad \mathrm{m}_{\mathrm{i}}=1, \quad i=J+1, \ldots, \mathrm{N} \text {. }
$$

Then, the left-preconditioned system is

$$
\tilde{A}_{N} U^{N}=\tilde{f}^{N} \text {, }
$$

where $\tilde{A}_{N}=M A_{N}$ and $\tilde{f}^{N}=M f^{N}$. The entries of $\tilde{A}_{N}$ are denoted by $\tilde{a}_{i j}$, and the nonzero ones are

$$
\tilde{l}_{i}:=\tilde{a}_{i, i-1}= \begin{cases}-\frac{\varepsilon}{h_{i} H}, & 1 \leqslant i \leqslant J, \\ -\frac{\varepsilon}{H^{2}}, & J+1 \leqslant i \leqslant N-1,\end{cases}
$$




$$
\tilde{r}_{i}:=\tilde{a}_{i, i+1}= \begin{cases}-\frac{\varepsilon}{h_{i+1} H}-\frac{b_{i} \bar{h}_{i}}{h_{i+1} H}, & 1 \leqslant i \leqslant J-1, \\ -\frac{\varepsilon}{H^{2}}-\frac{b_{b_{i}} h_{i}}{H^{2}}, & i=J, \\ -\frac{\varepsilon}{H^{2}}-\frac{b_{i}}{H}, & J+1 \leqslant i \leqslant N-1,\end{cases}
$$

and

$$
\tilde{\mathrm{d}}_{i}:=\tilde{\mathrm{a}}_{i i}= \begin{cases}1, & i=0, \\ -\tilde{\mathrm{l}}_{i}-\tilde{r}_{i}+c_{i} \frac{\bar{h}_{i}}{H}, & 1 \leqslant i \leqslant J, \\ -\tilde{\mathrm{l}}_{i}-\tilde{r}_{i}+c_{i}, & J+1 \leqslant i \leqslant N-1, \\ 1, & i=N .\end{cases}
$$

In order to show the $\varepsilon$-uniform stability of the preconditioned discretization (13), we need to show that $\tilde{A}_{N}$ is an M-matrix. We first derive a technical lemma that results from the special structure of the Bakhvalov-type mesh.

Lemma 2. Let $\beta>2$ and $\Delta_{i}:=\phi_{\mathrm{B}}\left(t_{\mathrm{i}}\right)-\phi_{\mathrm{B}}\left(\mathrm{t}_{\mathrm{i}-1}\right)$. Then, there exist a sufficiently large $\mathrm{N}_{0}$, and a positive constant $\delta$ independent of both $\varepsilon$ and $\mathrm{N}$, such that for all $\mathrm{N} \geqslant \mathrm{N}_{0}$, we have

$$
S_{i}:=\frac{\beta}{2}\left(1+\frac{\Delta_{i}}{\Delta_{i+1}}\right)-\left(\frac{1}{\Delta_{i}}-\frac{1}{\Delta_{i+1}}\right) \geqslant \delta>0, \quad i=1, \ldots, J-1 .
$$

Proof: From the assumption $\beta>2$ there exists a constant $\delta>0$, independent of $\varepsilon$ and $N$, such that $\beta / 2 \geqslant 1+\delta$. Then $S_{i}$, for $1 \leqslant i \leqslant J-1$, can be bounded from below as

$$
\begin{aligned}
S_{i} & \geqslant(1+\delta)\left(1+\frac{\Delta_{i}}{\Delta_{i+1}}\right)-\left(\frac{1}{\Delta_{i}}-\frac{1}{\Delta_{i+1}}\right) \\
& \geqslant\left(1+\frac{\Delta_{i}}{\Delta_{i+1}}+\frac{1}{\Delta_{i+1}}-\frac{1}{\Delta_{i}}\right)+\delta=\frac{\Delta_{i} \Delta_{i+1}+\Delta_{i}^{2}+\Delta_{i}-\Delta_{i+1}}{\Delta_{i} \Delta_{i+1}}+\delta .
\end{aligned}
$$

The assertion in (14) is proven if we can show that the numerator $\Delta_{i} \Delta_{i+1}+$ $\Delta_{i}^{2}+\Delta_{i}-\Delta_{i+1} \geqslant 0$. By direct computations, we get

$$
\Delta_{i}=\ln \left(1+\frac{1}{N /(2(1-\varepsilon))-i}\right)=\ln (1+z),
$$


where

$$
z:=[N /(2(1-\varepsilon))-i]^{-1} .
$$

On the other hand, in a different form

$$
\begin{aligned}
\Delta_{i+1} & =\ln \left(\frac{1-2(1-\varepsilon) t_{i}}{1-2(1-\varepsilon) t_{i+1}}\right) \\
& =\ln \left(1-\frac{1}{N /(2(1-\varepsilon))-i}\right)^{-1}=\ln \left(\frac{1}{1-z}\right) .
\end{aligned}
$$

Then,

$$
\Delta_{i} \Delta_{i+1}+\Delta_{i}^{2}+\Delta_{i}-\Delta_{i+1}=\ln (1+z)\left(\ln \frac{1+z}{1-z}+1\right)+\ln (1-z)=: g(z) .
$$

The function $\mathrm{g}(z)$ is defined for $z \in(-1,1)$. Its two roots are $z_{*}:=0$ and an irrational one denoted by $z^{*}$. We investigate $g(z)$ numerically on the interval $[0,0.98]$ using Maple - a computer algebra system - and its graph on this interval is plotted in Figure 2 which shows $g(z) \geqslant 0$ for all values of $z$ in between its two roots, $z_{*}$ and $z^{*}$. Additionally, the second root $z^{*}$ is numerically approximated as $z^{*} \approx 0.971$.

We now show that for each $\varepsilon$ there exists a sufficiently large $N$ such that $g(z)>0$. That is, by the definition (15) of $z$, the lower and upper bounds of $z$ are

$$
\begin{aligned}
0<\frac{1}{\mathrm{~N} /(2(1-\varepsilon))} \leqslant z & \leqslant \frac{1}{\mathrm{~N} /(2(1-\varepsilon))-(\mathrm{J}-1)} \\
& =\frac{1}{\mathrm{~N} /(2(1-\varepsilon))-(\mathrm{N} / 2-1)}=\frac{1}{1+\frac{\mathrm{N}}{2}\left(\frac{1}{1-\varepsilon}-1\right)} \\
& \leqslant \frac{1}{1+\mathrm{N}_{0} \eta}<z^{*},
\end{aligned}
$$

with $\eta:=\frac{1}{2}\left(\frac{1}{1-\varepsilon}-1\right)$. We can choose a sufficiently large integer $N_{0}$ to guarantee that $\frac{1}{1+N_{0} \eta} \leqslant 0.971<z^{*}$ because $\eta>0$ for all $\varepsilon<1$. 


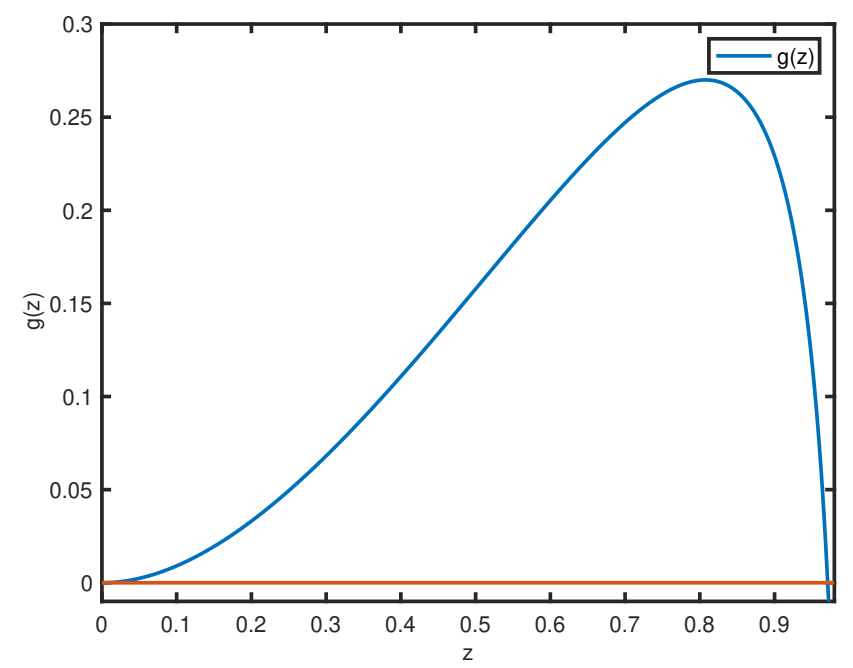

Figure 2: Graph of the function $\mathrm{g}(z)$ for $z \in[0,0.98]$.

Remark 3. In Lemma 2, the technical assumption $\beta>2$ can be relaxed to $\beta>0$ by having a user-chosen positive parameter $a$ in the definition of the graded mesh points $x_{i}$ in (6); that is, set $x_{i}=a \varepsilon \phi_{B}\left(t_{i}\right)$ for $i=0,1, \ldots, J$.

Lemma 4. Under the assumptions of Lemma 2 , the matrix $\tilde{A}_{\mathrm{N}}$ of the system (13) satisfies $\left\|\tilde{A}_{\mathrm{N}}^{-1}\right\| \leqslant \mathrm{C}$.

Proof: We only outline the essential calculation here. The detailed proof can be found elsewhere $[6,14,15]$. We construct a vector $v=\left[v_{0}, v_{1}, \ldots, v_{N}\right]^{\top}$ with elements

$$
v_{i}=\alpha-H i+\lambda \min \left\{(1+\rho)^{J-i}, 1\right\}, \quad i=0,1, \ldots, N,
$$

where $\alpha$ and $\lambda$ are appropriately chosen positive constants and $\rho=\beta \mathrm{H} / \varepsilon$. The following argument is to prove the condition: $\tilde{l}_{i} v_{i-1}+\tilde{d}_{i} v_{i}+\tilde{r}_{i} v_{i+1} \geqslant \delta_{*}$, $i=1,2, \ldots, N-1$, where $\delta_{*}$ is a positive constant independent of both 
$\varepsilon$ and $N$. Firstly, for $i=1, \ldots, J-1$, we have

$$
\begin{aligned}
\tilde{l}_{i} v_{i-1}+\tilde{d}_{i} v_{i}+\tilde{r}_{i} v_{i+1} & =\frac{\bar{h}_{i}}{H} c_{i} v_{i}-\frac{\varepsilon}{h_{i}}+\frac{\varepsilon}{h_{i+1}}+\frac{b_{i} \bar{h}_{i}}{h_{i+1}} \\
& \geqslant-\left(\frac{\varepsilon}{h_{i}}-\frac{\varepsilon}{h_{i+1}}\right)+\frac{b_{i}}{2}+\frac{b_{i} h_{i}}{2 h_{i+1}} \\
& =S_{i} \geqslant \delta>0,
\end{aligned}
$$

by Lemma 2. Secondly, when $i=J$,

$$
\begin{aligned}
\tilde{\mathrm{l}}_{J} v_{J-1}+\tilde{\mathrm{d}}_{J} v_{J}+\tilde{r}_{J} v_{J+1} & =\frac{\overline{\mathrm{h}}_{J}}{\mathrm{H}} c_{J} v_{J}+\tilde{\mathrm{l}}_{J} \mathrm{H}-\tilde{\mathrm{r}}_{J}\left(\mathrm{H}+\frac{\lambda \rho}{1+\rho}\right) \\
& \geqslant-\tilde{\mathrm{r}}_{J} \frac{\lambda \rho}{1+\rho}+\left(\tilde{\mathrm{l}}_{J}-\tilde{\mathrm{r}}_{J}\right) \mathrm{H} \\
& \geqslant\left(\frac{2 \varepsilon+\mathrm{b}_{J}\left(\mathrm{~h}_{J}+\mathrm{H}\right)}{2 \mathrm{H}^{2}}\right) \frac{\lambda \beta \mathrm{H}}{\varepsilon+\beta \mathrm{H}}-\frac{\varepsilon}{\mathrm{h}_{J}}+\frac{\mathrm{b}_{J}}{2} \\
& \geqslant \frac{\lambda \beta}{2 \mathrm{H}}-\frac{\varepsilon}{\mathrm{h}_{J}}+\frac{\beta}{2} \geqslant \frac{\beta}{2},
\end{aligned}
$$

provided

$$
\frac{\lambda \beta}{2 \mathrm{H}}-\frac{\varepsilon}{h_{\mathrm{J}}} \geqslant 0 .
$$

We bound $h_{J}$ from below as follows:

$$
h_{J}=\varepsilon \int_{t_{J-1}}^{t_{J}} \phi_{B}^{\prime}(s) d s \geqslant \frac{\varepsilon}{N} \phi_{B}^{\prime}\left(t_{J-1}\right)=\frac{\varepsilon}{N} \frac{2(1-\varepsilon)}{1-2(1-\varepsilon) t_{J-1}} \geqslant \frac{2 \varepsilon\left(1-\varepsilon^{*}\right)}{N},
$$

where $0<\varepsilon \leqslant \varepsilon^{*}<1$. Therefore,

$$
\begin{aligned}
\frac{\lambda \beta}{2 \mathrm{H}}-\frac{\varepsilon}{h_{J}} & \geqslant \frac{\lambda \beta}{2 \mathrm{H}}-\frac{\varepsilon N}{2 \varepsilon\left(1-\varepsilon^{*}\right)}=\frac{\lambda \beta N}{4}-\frac{N}{2\left(1-\varepsilon^{*}\right)} \\
& \geqslant N\left(\frac{\lambda \beta}{4}-\frac{1}{2\left(1-\varepsilon^{*}\right)}\right) \geqslant 0
\end{aligned}
$$


where we use $\mathrm{N}^{-1} \leqslant \mathrm{H} \leqslant 2 \mathrm{~N}^{-1}$, and we choose $\lambda \geqslant \frac{2}{\beta\left(1-\varepsilon^{*}\right)}$. Finally, when $i=J+1, \ldots, N-1$, we have

$$
\begin{aligned}
\tilde{l}_{i} v_{i-1}+\tilde{d}_{i} v_{i}+\tilde{r}_{i} v_{i+1}= & c_{i} v_{i}+\tilde{l}_{i} H-\tilde{r}_{i} H+\tilde{l}_{i}\left[\frac{\lambda}{(1+\rho)^{i-1-J}}-\frac{\lambda}{(1+\rho)^{i-J}}\right] \\
& +\tilde{r}_{i}\left[\frac{\lambda}{(1+\rho)^{i+1-J}}-\frac{\lambda}{(1+\rho)^{i-J}}\right] \\
\geqslant & b_{i}+\frac{\lambda}{(1+\rho)^{i+1-J}}\left[\tilde{r}_{i}-\tilde{r}_{i}(1+\rho)+\tilde{l}_{i}(1+\rho)^{2}\right. \\
& \left.-\tilde{l}_{i}(1+\rho)\right] \\
= & b_{i}+\frac{\lambda \rho}{(1+\rho)^{i+1-J}}\left[\tilde{l}_{i}-\tilde{r}_{i}+\tilde{l}_{i} \rho\right] \\
\geqslant & \beta+\frac{\lambda \rho}{(1+\rho)^{i+1-J}}\left[\frac{b_{i}}{H}-\frac{\beta}{H}\right] \geqslant \beta .
\end{aligned}
$$

By choosing $\delta_{*}=\min \{\delta, \beta / 2\}$ we complete the proof.

\section{$4 \varepsilon$-uniform consistency and convergence}

Let $\tau_{i}[g]=\mathcal{L}^{N} g_{i}-(\mathcal{L} g)_{i}, i=1,2, \ldots, N-1$, for any $C^{2}(I)$-function $g$. In particular, $\tau_{i}[u]$ is the truncation error of the finite-difference operator $\mathcal{L}^{\mathrm{N}}$ and

$$
\tau_{i}[u]=\mathcal{L}^{N} u_{i}-\mathcal{L}^{N} u_{i}^{N}=\mathcal{L}^{N}\left(u-u^{N}\right)_{i}=\left[A_{N}\left(u^{N}-u^{N}\right)\right]_{i},
$$

whereas the preconditioned consistency error is

$$
\tilde{\tau}_{i}[u]= \begin{cases}\frac{\bar{h}_{i}}{H} \tau_{i}[u], & i=1, \ldots, J, \\ \tau_{i}[u], & i=J+1, \ldots, N-1 .\end{cases}
$$

By Taylor's expansion

$$
\left|\tau_{i}[u]\right| \leqslant C h_{i+1}\left(\varepsilon\left\|u^{\prime \prime \prime}\right\|_{i}+\left\|u^{\prime \prime}\right\|_{i}\right),
$$


where $\|g\|_{i}:=\max _{x_{i-1} \leqslant x \leqslant x_{i+1}}|g(x)|$ for any $g \in C(I)$.

Theorem 5. Let $\beta>2$. The preconditioned consistency error $\tilde{\tau}_{i}[u]$ is bounded uniformly in $\varepsilon:\left|\tilde{\tau}_{i}[u]\right| \leqslant C N^{-1}, i=1, \ldots, N-1$.

Proof: We use the decomposition (2) to get

$$
\tilde{\tau}_{i}[u]=\tilde{\tau}_{i}[s]+\tilde{\tau}_{i}[y], \quad i=1, \ldots, N-1,
$$

and the estimates (3) to bound the terms on the right hand side separately. For the smooth part of the solution, $\left|\tilde{\tau}_{i}[s]\right| \leqslant \mathrm{CN}^{-1}, 1 \leqslant i \leqslant N-1$, due to (8) and $\mathrm{H} \leqslant 2 \mathrm{~N}^{-1}$. Then, for the singular component, we need to show that $\left|\tilde{\tau}_{i}[y]\right| \leqslant C N^{-1}, i=1, \ldots, N-1$.

We divide the proof into cases regarding the indices $i$. For $i \geqslant J+1$, we apply (17) to $y$ and use the derivative estimate (3). Then,

$$
\left|\tilde{\tau}_{i}[y]\right|=\left|\tau_{i}[y]\right| \leqslant C h_{i+1}\left(\varepsilon\left\|y^{\prime \prime \prime}\right\|_{i}+\left\|y^{\prime \prime}\right\|_{i}\right) \leqslant C N^{-1} \mathcal{E}^{-2} e^{-\beta x_{j} / \varepsilon} \leqslant C N^{-1},
$$

where use (11) and $\beta>2$ in the last inequality.

For $i=J$, we have $\left|\tilde{\tau}_{J}[y]\right|=\frac{\bar{h}_{I}}{H}\left|\tau_{J}[y]\right| \leqslant C\left|\tau_{J}[y]\right|$, so we bound $\left|\tau_{J}[y]\right|$ directly by considering two cases: $\varepsilon \leqslant N^{-1}$ and $\varepsilon>N^{-1}$. First, when $i=J$ and $\varepsilon \leqslant N^{-1}$ we use the truncation error estimate in the form of $\tau_{i}[y]=\mathcal{L}^{N} y$, which is valid because of (4). Thus, we have

$$
\left|\tau_{i}[y]\right| \leqslant P_{i}+Q_{i}+R_{i}, \quad P_{i}=\varepsilon\left|D^{\prime \prime} y_{i}\right|, \quad Q_{i}=b_{i}\left|D^{\prime} y_{i}\right|, \quad \text { and } \quad R_{i}=c_{i}\left|y_{i}\right| .
$$

We bound $P_{\mathrm{J}}$ from above as follows. Since $\bar{h}_{\mathrm{J}} \geqslant h_{\mathrm{J}+1} / 2 \geqslant C N^{-1}$, we get $\overline{\mathrm{h}}_{\mathrm{J}}^{-1} \leqslant \mathrm{CN}$ and invoking $(10)$,

$$
\mathrm{P}_{\mathrm{J}} \leqslant \mathrm{Ch}_{\mathrm{J}}^{-1} e^{-\beta x_{\mathrm{J}-1 / \varepsilon}} \leqslant \mathrm{CN}\left(\varepsilon+2 \mathrm{~N}^{-1}\right)^{\beta} \leqslant \mathrm{CN}^{-1} \text {. }
$$

Analogous arguments can be applied to $Q_{J}$ and $R_{J}$ to imply that $\left|\tau_{J}[y]\right| \leqslant$ $\mathrm{CN}^{-1}$. 
Second, when $\boldsymbol{i}=J$ and $\varepsilon>N^{-1}$ we get that $h_{J} \leqslant C \varepsilon$ because of (8). Therefore, similarly to (18),

$$
\left|\tau_{\mathrm{J}}[y]\right| \leqslant \mathrm{CN}^{-1} \mathcal{E}^{-2} e^{-\beta x_{\mathrm{J}-1} / \varepsilon} \leqslant \mathrm{CN}^{-1} \mathcal{E}^{-2} e^{-\beta x_{\mathrm{J}} / \varepsilon} \leqslant \mathrm{CN}^{-1} .
$$

For $i \leqslant J-1$ we prove that

$$
\left|\tilde{\tau}_{i}[u]\right| \leqslant C N^{-1} \text { when }\left\{\begin{array}{l}
i \leqslant J-2, \\
i=J-1 \text { and } h_{J} \leqslant \varepsilon \\
i=J-1 \text { and } h_{J}>\varepsilon .
\end{array}\right.
$$

We first prove the first two cases of $(20)$. For $i \leqslant J-1$ we have $h_{i} \leqslant \varepsilon$ because of $(9)$ and $h_{J} \leqslant \varepsilon$ by the assumption. Hence,

$$
\begin{aligned}
\left|\tilde{\tau}_{i}[y]\right| & \leqslant C \frac{\bar{h}_{i}}{H} h_{i+1}\left(\varepsilon\left\|y^{\prime \prime \prime}\right\|_{i}+\left\|y^{\prime \prime}\right\|_{i}\right) \leqslant N^{-1}\left[\varepsilon \phi_{B}^{\prime}\left(t_{i+1}\right)\right]^{2}\left(\varepsilon^{-2} e^{-\beta x_{i-1} / \varepsilon}\right) \\
& \leqslant C N^{-1}\left[\phi_{B}^{\prime}\left(t_{i+1}\right)\right]^{2} e^{-\beta x_{i+1} / \varepsilon} \leqslant C N^{-1}\left[1-2(1-\varepsilon) t_{i+1}\right]^{\beta-2} \leqslant C N^{-1} .
\end{aligned}
$$

Lastly, when $i=J-1$ and $h_{J}>\varepsilon$, this means that $\max \left\{\varepsilon, h_{J}\right\}=h_{J}$. Then, $\varepsilon \leqslant \mathrm{CN}^{-1}$, again because of (8), and similarly to (19) we use (10) to get

$$
\begin{aligned}
\left|\tilde{\tau}_{J-1}[y]\right| & \leqslant C \frac{\bar{h}_{J-1}}{\mathrm{H}}\left(P_{J-1}+Q_{J-1}+R_{J-1}\right) \\
& \leqslant C \frac{\bar{h}_{J-1}}{\mathrm{H}}\left[\frac{1}{\bar{h}_{J-1}} \varepsilon \cdot 2\left\|y^{\prime}\right\|_{J-1}+\frac{1}{h_{J}}\|y\|_{J-1}+e^{-\beta x_{J-2} / \varepsilon}\right] \\
& \leqslant C N e^{-\beta x_{J-2} / \varepsilon} \leqslant C N e^{-\beta x_{J-1} / \varepsilon} \leqslant C N\left(\varepsilon+2 N^{-1}\right)^{\beta} \leqslant C N^{-1} .
\end{aligned}
$$

Combining Lemma 4 ( $\varepsilon$-uniform stability) and Theorem 5 ( $\varepsilon$-uniform consistency), we arrive the uniform convergence result.

Theorem 6. On the Bakhvalov-type mesh defined in (6) and (7), the upwind difference scheme applied to the problem (1) is first-order uniformly convergent: $\left|u_{i}-u_{i}^{N}\right| \leqslant C N^{-1}, 0 \leqslant i \leqslant N$. 
Acknowledgements The authors thank the referees for their careful reading and valuable corrections and suggestions. VQM thanks Thu Dau Mot University for financial support.

\section{References}

[1] N. S. Bakhvalov. "The optimization of methods of solving boundary value problems with a boundary layer". In: USSR Comput. Math. Math. Phys. 9.4 (1969), pp. 139-166. DOI: 10.1016/0041-5553(69)90038-X. (Cit. on p. C147).

[2] I. P. Boglaev. "Approximate solution of a non-linear boundary value problem with a small parameter for the highest-order differential". In: USSR Comput. Math. Math. Phys. 24.6 (1984), pp. 30-35. DOI: 10.1016/0041-5553(84)90005-3. (Cit. on p. C150).

[3] T. Linß. Layer-adapted meshes for reaction-convection-diffusion problems. Vol. 1985. Lecture Notes in Mathematics. Springer-Verlag, 2010. DOI: 10.1007/978-3-642-05134-0. (Cit. on pp. C147, C149).

[4] T. Linß, H.-G. Roos, and R. Vulanović. "Uniform pointwise convergence on Shishkin-type meshes for quasi-linear convection-diffusion problems". In: SIAM J. Numer. Anal. 38.3 (2000), pp. 897-912. DOI: 10.1137/S0036142999355957. (Cit. on p. C148).

[5] V. D. Liseikin. Layer resolving grids and transformations for singular perturbation problems. De Gruyter, 2001. DOI: 10.1515/9783110941944. (Cit. on p. C148).

[6] T. A. Nhan, M. Stynes, and R. Vulanović. "Optimal uniform-convergence results for convection-diffusion problems in one dimension using preconditioning". In: J. Comput. Appl. Math. 338 (2018), pp. 227-238. DOI: 10.1016/j.cam.2018.02.012. (Cit. on pp. C148, C155). 
[7] T. A. Nhan and R. Vulanović. "Analysis of the truncation error and barrier-function technique for a Bakhvalov-type mesh". In: Electron. Trans. Numer. Anal. 51 (2019), pp. 315-330. DOI: 10.1553/etna_vol51s315 (cit. on p. C148).

[8] T. A. Nhan and R. Vulanović. "The Bakhvalov mesh: a complete finite-difference analysis of two-dimensional singularly perturbed convection-diffusion problems". In: Numer. Alg. 87 (2021), pp. 203-221. DOI: $10.1007 / \mathrm{s} 11075-020-00964-\mathrm{z}$ (cit. on pp. C148, C150).

[9] H.-G. Roos and T. Linß. "Sufficient conditions for uniform convergence on layer-adapted grids". In: Computing 63.1 (1999), pp. 27-45. DOI: 10.1007/s006070050049. (Cit. on p. C150).

[10] H.-G. Roos and M. Stynes. "Some open questions in the numerical analysis of singularly perturbed differential equations". In: Comput. Meth. Appl. Math. 15.4 (2015), pp. 531-550. DOI: 10.1515/cmam-2015-0011. (Cit. on p. C147).

[11] H.-G. Roos, M. Stynes, and L. Tobiska. Robust numerical methods for singularly perturbed differential equations. Vol. 24. Springer Series in Computational Mathematics. Springer-Verlag, 2008. DOI: 10.1007/978-3-540-34467-4 (cit. on p. C147).

[12] G. I. Shishkin. "A difference scheme for a singularly perturbed equation of parabolic type with discontinuous boundary conditions". In: USSR Comput. Math. Math. Phys. 28.6 (1988), pp. 32-41. DOI: 10.1016/0041-5553(88)90039-0. (Cit. on p. C147).

[13] M. Stynes. "Steady-state convection-diffusion problems". In: Acta Numer. 14 (2005), pp. 445-508. DOI: 10.1017/S0962492904000261. (Cit. on p. C147).

[14] R. Vulanović and T. A. Nhan. "Uniform convergence via preconditioning". In: Int. J. Numer. Anal. Model. Ser. B 5.4 (2014), pp. 347-356. URL: https://www.globalsci.org/intro/article_detail/ijnamb/239.html (cit. on pp. C148, C155). 
[15] R. Vulanović and T. A. Nhan. "Robust hybrid schemes of higher order for singularly perturbed convection-diffusion problems". In: Appl. Math. Comput. 386 (2020), p. 125495. DOI: 10.1016/j.amc.2020.125495. (Cit. on pp. C148, C155).

\section{Author addresses}

1. Thái Anh Nhan, Department of Mathematics and Science, Holy Names University, 3500 Mountain Blvd., Oakland, CA 94619, USA. mailto:nhan@hnu.edu orcid:0000-0001-9779-2371

2. Vinh Quang Mai, Department of Mathematics, Thu Dau Mot University, Thu Dau Mot City, Binhduong, Vietnam.

mailto:vinhmq@tdmu .edu.vn orcid:0000-0001-7434-5843 